\title{
Optimal convex combination bounds of geometric and Neuman means for Toader-type mean
}

Yue-Ying Yang ${ }^{1 *}$ (D) and Wei-Mao Qian²

"Correspondence:

yyy1008hz@163.com

'School of Mechanical and

Electrical Engineering, Huzhou Vocational \& Technical College,

Huzhou, 313000, China

Full list of author information is

available at the end of the article

\begin{abstract}
In this paper, we prove that the double inequalities

$$
\begin{aligned}
& \alpha N_{Q A}(a, b)+(1-\alpha) G(a, b)<\operatorname{TD}[A(a, b), G(a, b)]<\beta N_{Q A}(a, b)+(1-\beta) G(a, b), \\
& \lambda N_{A Q}(a, b)+(1-\lambda) G(a, b)<\operatorname{TD}[A(a, b), G(a, b)]<\mu N_{A Q}(a, b)+(1-\mu) G(a, b)
\end{aligned}
$$

hold for all $a, b>0$ with $a \neq b$ if and only if $\alpha \leq 3 / 8$,

$\beta \geq 4 /[\pi(\log (1+\sqrt{2})+\sqrt{2})]=0.5546 \cdots, \lambda \leq 3 / 10$ and

$\mu \geq 8 /[\pi(\pi+2)]=0.4952 \cdots$, where $\operatorname{TD}(a, b), G(a, b), A(a, b)$ and $N_{Q A}(a, b), N_{A Q}(a, b)$ are the Toader, geometric, arithmetic and two Neuman means of $a$ and $b$, respectively.
\end{abstract}

MSC: 26E60; 33E05

Keywords: Toader mean; geometric mean; Neuman mean

\section{Introduction}

For $x, y, z \geq 0$ with $x y+x z+y z \neq 0$ and $r \in(0,1)$, the symmetric integrals $R_{F}(x, y, z)$ and $R_{G}(x, y, z)[1]$ of the first and second kinds, and the complete elliptic integrals $\mathcal{K}(r)$ and $\mathcal{E}(r)$ of the first and second kinds are defined by

$$
\begin{aligned}
& R_{F}(x, y, z)=\frac{1}{2} \int_{0}^{\infty}[(t+x)(t+y)(t+z)]^{-1 / 2} d t \\
& R_{G}(x, y, z)=\frac{1}{4} \int_{0}^{\infty}[(t+x)(t+y)(t+z)]^{-1 / 2}\left(\frac{x}{t+x}+\frac{y}{t+y}+\frac{z}{t+z}\right) t d t \\
& \mathcal{K}(r)=\int_{0}^{\pi / 2}\left[1-r^{2} \sin ^{2}(t)\right]^{-1 / 2} d t, \quad \mathcal{E}(r)=\int_{0}^{\pi / 2}\left[1-r^{2} \sin ^{2}(t)\right]^{1 / 2} d t
\end{aligned}
$$

respectively.

The well-known identities

$$
\mathcal{K}(r)=R_{F}\left(0,1-r^{2}, 1\right), \quad \mathcal{E}(r)=2 R_{G}\left(0,1-r^{2}, 1\right)
$$

were established by Carlson in [1].

(c) The Author(s) 2017. This article is distributed under the terms of the Creative Commons Attribution 4.0 International License (http://creativecommons.org/licenses/by/4.0/), which permits unrestricted use, distribution, and reproduction in any medium, provided you give appropriate credit to the original author(s) and the source, provide a link to the Creative Commons license, and indicate if changes were made. 
Let $a, b>0$ with $a \neq b$. Then the Toader mean $T D(a, b)[2]$ and the Schwab-Borchardt mean $S B(a, b)[3-5]$ are respectively defined by

$$
\begin{aligned}
T D(a, b) & =\frac{2}{\pi} \int_{0}^{\pi / 2} \sqrt{a^{2} \cos ^{2}(t)+b^{2} \sin ^{2}(t)} d t \\
& = \begin{cases}2 a \mathcal{E}\left(\sqrt{1-(b / a)^{2}}\right) / \pi, & a>b, \\
2 b \mathcal{E}\left(\sqrt{1-(a / b)^{2}}\right) / \pi, & a<b,\end{cases}
\end{aligned}
$$

and

$$
S B(a, b)= \begin{cases}\frac{\sqrt{b^{2}-a^{2}}}{\cos ^{-1}(a / b)}, & a<b, \\ \frac{\sqrt{a^{2}-b^{2}}}{\cosh ^{-1}(a / b)}, & a>b,\end{cases}
$$

where $\cos ^{-1}(x)$ and $\cosh ^{-1}(x)=\log \left(x+\sqrt{x^{2}-1}\right)$ are the inverse cosine and inverse hyperbolic cosine functions, respectively.

Very recently, Neuman [6] introduced the Neuman mean $N(a, b)$ of the second kind as follows:

$$
N(a, b)=\frac{1}{2}\left[a+\frac{b^{2}}{S B(a, b)}\right]
$$

It is well known that the Toader mean $T D(a, b)$, the Schwab-Borchardt mean $S B(a, b)$ and the Neuman mean of the second kind $N(a, b)$ satisfy the identities (see [6, 7])

$$
\begin{aligned}
T D(a, b) & =\frac{4}{\pi} R_{G}\left(a^{2}, b^{2}, 0\right) \\
& =\frac{1}{\pi} \int_{0}^{\infty}\left[\left(t+a^{2}\right)\left(t+b^{2}\right)\right]^{-1 / 2}\left(\frac{a^{2}}{t+a^{2}}+\frac{b^{2}}{t+b^{2}}\right) t d t, \\
S B(a, b) & =1 / R_{F}\left(a^{2}, b^{2}, b^{2}\right) \\
& =2 / \int_{0}^{\infty}\left[\left(t+a^{2}\right)\left(t+b^{2}\right)\left(t+b^{2}\right)\right]^{-1 / 2} d t, \\
N(a, b) & =R_{G}\left(a^{2}, b^{2}, b^{2}\right) \\
& =\frac{1}{4} \int_{0}^{\infty}\left[\left(t+a^{2}\right)\left(t+b^{2}\right)\left(t+b^{2}\right)\right]^{-1 / 2}\left(\frac{a^{2}}{t+a^{2}}+\frac{b^{2}}{t+b^{2}}+\frac{b^{2}}{t+b^{2}}\right) t d t .
\end{aligned}
$$

Let $p \in \mathbb{R}$ and $a, b>0$. Then the $p$ th power mean $M_{p}(a, b)$ is defined by

$$
M_{p}(a, b)=\left[\left(a^{p}+b^{p}\right) / 2\right]^{1 / p}(p \neq 0), \quad M_{0}(a, b)=\sqrt{a b}
$$

We clearly see that $M_{p}(a, b)$ is symmetric and homogeneous of degree one with respect to $a$ and $b$, strictly increasing with respect to $p \in \mathbb{R}$ for fixed $a, b>0$ with $a \neq b$, and the inequalities

$$
G(a, b)=M_{0}(a, b)<A(a, b)=M_{1}(a, b)<Q(a, b)=M_{2}(a, b)
$$


hold for $a, b>0$ with $a \neq b$, where $G(a, b)=\sqrt{a b}, A(a, b)=(a+b) / 2$ and $Q(a, b)=$ $\sqrt{\left(a^{2}+b^{2}\right) / 2}$ are the geometric, arithmetic and quadratic means of $a$ and $b$, respectively. In [6], Neuman presented the explicit formula for $N_{Q A}(a, b) \equiv N[Q(a, b), A(a, b)]$ and $N_{A Q}(a, b) \equiv N[A(a, b), Q(a, b)]$ as follows:

$$
\begin{aligned}
& N_{Q A}(a, b)=\frac{1}{2} A(a, b)\left[\sqrt{1+v^{2}}+\frac{\sinh ^{-1}(v)}{v}\right], \\
& N_{A Q}(a, b)=\frac{1}{2} A(a, b)\left[1+\left(1+v^{2}\right) \frac{\tan ^{-1}(v)}{v}\right]
\end{aligned}
$$

and proved that the inequalities

$$
A(a, b)<N_{Q A}(a, b)<N_{A Q}(a, b)<Q(a, b)
$$

hold for $a, b>0$ with $a \neq b$, where $v=(a-b) /(a+b)$.

Recently, the Toader mean has been the subject of intensive research. In particular, many remarkable inequalities for Toader mean and other related means can be found in the literature [8-41].

In [42], Vuorinen conjectured that

$$
T D(a, b)>M_{3 / 2}(a, b)
$$

for all $a, b>0$ with $a \neq b$. This conjecture was proved by Qiu and Shen [43], and Barnard et al. [44], respectively, and Alzer and Qiu [45] presented the best possible upper power mean bound for the Toader mean as follows:

$$
T D(a, b)<M_{\log 2 / \log (\pi / 2)}(a, b)
$$

for all $a, b>0$ with $a \neq b$.

Li, Qian and Chu [46] proved that the inequality

$$
\alpha N_{A Q}(a, b)+(1-\alpha) A(a, b)<T D(a, b)<\beta N_{A Q}(a, b)+(1-\beta) A(a, b)
$$

holds for all $a, b>0$ with $a \neq b$ if and only if $\alpha \leq 3 / 4$ and $\beta \geq 4(4-\pi) /[\pi(\pi-2)]=$ $0.9573 \cdots$.

Note that

$$
G(a, b)<T D[A(a, b), G(a, b)]<A(a, b)
$$

for all $a, b>0$ with $a \neq b$.

From inequalities (1.5) and (1.6) we clearly see that

$$
G(a, b)<T D[A(a, b), G(a, b)]<N_{Q A}(a, b)<N_{A Q}(a, b)
$$

for all $a, b>0$ with $a \neq b$. 
The main purpose of this paper is to find the greatest values $\alpha, \lambda$ and the least values $\beta$, $\mu$ such that the double inequalities

$$
\begin{aligned}
& \alpha N_{Q A}(a, b)+(1-\alpha) G(a, b)<T D[A(a, b), G(a, b)]<\beta N_{Q A}(a, b)+(1-\beta) G(a, b), \\
& \lambda N_{A Q}(a, b)+(1-\lambda) G(a, b)<T D[A(a, b), G(a, b)]<\mu N_{A Q}(a, b)+(1-\mu) G(a, b)
\end{aligned}
$$

hold for all $a, b>0$ with $a \neq b$. As applications, we get two new bounds for the complete elliptic integral of the second kind in terms of elementary functions.

\section{Lemmas}

In order to prove our main results, we need several lemmas, which we present in this section.

For $r \in(0,1)$, we clearly see that

$$
\mathcal{K}\left(0^{+}\right)=\mathcal{E}\left(0^{+}\right)=\pi / 2, \quad \mathcal{K}\left(1^{-}\right)=+\infty, \quad \mathcal{E}\left(1^{-}\right)=1,
$$

and $\mathcal{K}(r)$ and $\mathcal{E}(r)$ satisfy the formulas (see[21], Appendix E, pp.474-475)

$$
\begin{aligned}
& \frac{d \mathcal{K}(r)}{d r}=\frac{\mathcal{E}(r)-\left(1-r^{2}\right) \mathcal{K}(r)}{r\left(1-r^{2}\right)}, \quad \frac{d \mathcal{E}(r)}{d r}=\frac{\mathcal{E}(r)-\mathcal{K}(r)}{r}, \\
& \frac{d[\mathcal{E}(r)-\mathcal{K}(r)]}{d r}=-\frac{r \mathcal{E}(r)}{1-r^{2}} .
\end{aligned}
$$

Lemma 2.1 (see [21], Theorem 1.25) For $-\infty<a<b<+\infty$, let $f, g:[a, b] \rightarrow \mathbb{R}$ be continuous on $[a, b]$ and differentiable on $(a, b)$, and $g^{\prime}(x) \neq 0$ on $(a, b)$. If $f^{\prime}(x) / g^{\prime}(x)$ is increasing (decreasing) on $(a, b)$, then so are

$$
\frac{f(x)-f(a)}{g(x)-g(a)} \text { and } \frac{f(x)-f(b)}{g(x)-g(b)}
$$

If $f^{\prime}(x) / g^{\prime}(x)$ is strictly monotone, then the monotonicity in the conclusion is also strict.

Lemma 2.2 (see [21], Theorem 3.21(1), Exercise 3.43(11) and Exercise 3.43(29))

(1) The function $r \mapsto\left[\mathcal{E}(r)-\left(1-r^{2}\right) \mathcal{K}(r)\right] / r^{2}$ is strictly increasing from $(0,1)$ onto $(\pi / 4,1)$;

(2) The function $r \mapsto[\mathcal{K}(r)-\mathcal{E}(r)] / r^{2}$ is strictly increasing from $(0,1)$ onto $(\pi / 4,+\infty)$;

(3) The function $r \mapsto\left[\left(2-r^{2}\right) \mathcal{K}(r)-2 \mathcal{E}(r)\right] / r^{4}$ is strictly increasing from $(0,1)$ onto $(\pi / 16,+\infty)$.

Lemma 2.3 The function $r \mapsto \varphi_{1}(r)=\left\{\frac{2}{\pi} \sqrt{1-r^{2}}[2 \mathcal{E}(r)-\mathcal{K}(r)]+2 r^{2}-1\right\} / r^{2}$ is strictly increasing from $(0,1)$ onto $(3 / 4,1)$.

Proof Simple computations lead to

$$
\begin{aligned}
& \varphi_{1}\left(0^{+}\right)=\frac{3}{4}, \quad \varphi_{1}\left(1^{-}\right)=1, \\
& \varphi_{1}^{\prime}(r)=\frac{2}{\pi r^{3}} \gamma_{1}(r),
\end{aligned}
$$


where

$$
\begin{aligned}
& \gamma_{1}(r)=\frac{\mathcal{K}(r)-3 \mathcal{E}(r)}{\sqrt{1-r^{2}}}+\pi, \\
& \gamma_{1}\left(0^{+}\right)=0, \\
& \gamma_{1}^{\prime}(r)=\frac{r^{3}}{\left(1-r^{2}\right)^{3 / 2}} \frac{\left(2-r^{2}\right) \mathcal{K}(r)-2 \mathcal{E}(r)}{r^{4}} .
\end{aligned}
$$

From (2.5) and Lemma 2.2(3) we get

$$
\gamma_{1}^{\prime}(r)>\frac{\pi r^{3}}{16\left(1-r^{2}\right)^{3 / 2}}>0
$$

Therefore, Lemma 2.3 follows easily from (2.1), (2.2), (2.4) and (2.6).

Lemma 2.4 The function $r \mapsto \varphi_{2}(r)=\left(2 r^{2}+\sqrt{1-r^{4}}-1\right) / r^{2}$ is strictly decreasing from $(0,1)$ onto $(1,2)$.

Proof It is easy to verify that

$$
\begin{aligned}
& \varphi_{2}\left(0^{+}\right)=2, \quad \varphi_{2}\left(1^{-}\right)=1, \\
& \varphi_{2}^{\prime}(r)=\frac{2\left(\sqrt{1-r^{4}}-1\right)}{r^{3} \sqrt{1-r^{4}}}<0
\end{aligned}
$$

for $r \in(0,1)$.

Therefore, Lemma 2.4 follows easily from (2.7) and (2.8).

Lemma 2.5 The function $r \mapsto \varphi_{3}(r)=\left[2 r^{2} \mathcal{K}(r)-5 \mathcal{E}(r)\right] / \sqrt{1-r^{2}}$ is strictly increasing from $(0,1)$ onto $(-5 \pi / 2,+\infty)$.

Proof It is not difficult to verify that

$$
\begin{aligned}
& \varphi_{3}\left(0^{+}\right)=-\frac{5}{2} \pi, \quad \varphi_{3}\left(1^{-}\right)=+\infty \\
& \varphi_{3}^{\prime}(r)=\frac{r}{\left(1-r^{2}\right)^{3 / 2}}\left[\left(5-3 r^{2}\right) \frac{\mathcal{K}(r)-\mathcal{E}(r)}{r^{2}}-\mathcal{E}(r)\right] .
\end{aligned}
$$

From (2.10) and Lemma 2.2(2) together with the monotonicity of $\mathcal{E}(r)$ on $(0,1)$ we clearly see that

$$
\varphi_{3}^{\prime}(r)>\frac{r}{\left(1-r^{2}\right)^{3 / 2}}\left[\left(5-3 r^{2}\right) \times \frac{\pi}{4}-\frac{\pi}{2}\right]=\frac{3 \pi}{4} \frac{r}{\sqrt{1-r^{2}}}>0
$$

for $r \in(0,1)$.

Therefore, Lemma 2.5 follows from (2.9) and (2.11).

Lemma 2.6 The function $r \mapsto \varphi_{4}(r)=\left\{\frac{2}{\pi} \sqrt{1-r^{2}}\left[2 \mathcal{E}(r)-\left(1+r^{2}\right) \mathcal{K}(r)\right]+3 r^{2}-1\right\} / r^{2}$ is strictly increasing from $(0,1)$ onto $(3 / 4,2)$. 
Proof Let $\phi_{1}(r)=\frac{2}{\pi} \sqrt{1-r^{2}}\left[2 \mathcal{E}(r)-\left(1+r^{2}\right) \mathcal{K}(r)\right]+3 r^{2}-1, \phi_{2}(r)=r^{2}$. Then simple computations give

$$
\begin{aligned}
& \phi_{1}\left(0^{+}\right)=\phi_{2}(0)=0, \quad \varphi_{4}(r)=\phi_{1}(r) / \phi_{2}(r), \\
& \varphi_{4}\left(1^{-}\right)=2, \\
& \frac{\phi_{1}^{\prime}(r)}{\phi_{2}^{\prime}(r)}=3+\frac{1}{\pi \sqrt{1-r^{2}}}\left[\frac{\mathcal{E}(r)-\left(1-r^{2}\right) \mathcal{K}(r)}{r^{2}}\right]+\frac{1}{\pi} \varphi_{3}(r) .
\end{aligned}
$$

It follows from Lemma 2.2(1), Lemma 2.5 and the function $r \mapsto \sqrt{1-r^{2}}$ strictly decreasing that $\phi_{1}^{\prime}(r) / \phi_{2}^{\prime}(r)$ is strictly increasing on $(0,1)$ and

$$
\varphi_{4}\left(0^{+}\right)=\lim _{r \rightarrow 0^{+}} \frac{\phi_{1}^{\prime}(r)}{\phi_{2}^{\prime}(r)}=\frac{3}{4} .
$$

Therefore, Lemma 2.6 follows from Lemma 2.1, (2.12), (2.13) and (2.15) together with the monotonicity of $\phi_{1}^{\prime}(r) / \phi_{2}^{\prime}(r)$.

Lemma 2.7 The function $\varphi_{5}(r)=\left[3 r^{2}+\sqrt{1-r^{2}}-1\right] / r^{2}$ is strictly decreasing from $(0,1)$ onto $(2,5 / 2)$.

Proof We clearly see that

$$
\begin{aligned}
& \varphi_{5}\left(0^{+}\right)=\frac{5}{2}, \quad \varphi_{5}\left(1^{-}\right)=2, \\
& \varphi_{5}^{\prime}(r)=-\frac{\left(1-\sqrt{1-r^{2}}\right)^{2}}{r^{3} \sqrt{1-r^{2}}}<0
\end{aligned}
$$

for $r \in(0,1)$.

Therefore, Lemma 2.7 follows easily from (2.16) and (2.17).

\section{Main results}

Theorem 3.1 The double inequality

$$
\alpha N_{Q A}(a, b)+(1-\alpha) G(a, b)<T D[A(a, b), G(a, b)]<\beta N_{Q A}(a, b)+(1-\beta) G(a, b)
$$

holds for all $a, b>0$ with $a \neq b$ if and only if $\alpha \leq 3 / 8$ and $\beta \geq 4 /[\pi(\log (1+\sqrt{2})+\sqrt{2})]=$ $0.5546 \cdots$.

Proof Since $G(a, b), T D(a, b)$ and $N_{Q A}(a, b)$ are symmetric and homogenous of degree 1 , without loss of generality, we assume that $a>b>0$ and let $r=(a-b) /(a+b) \in(0,1)$. Then (1.1)-(1.3) lead to

$$
\begin{aligned}
& T D[A(a, b), G(a, b)]=\frac{2}{\pi} A(a, b) \mathcal{E}(r), \\
& G(a, b)=A(a, b) \sqrt{1-r^{2}}, \quad N_{Q A}(a, b)=\frac{1}{2} A(a, b)\left[\sqrt{1+r^{2}}+\frac{\sinh ^{-1}(r)}{r}\right] .
\end{aligned}
$$


It follows from (3.2)-(3.3) that

$$
\begin{aligned}
& \begin{array}{c}
\frac{T}{[}[A(a, b), G(a, b)]-G(a, b) \\
N_{Q A}(a, b)-G(a, b)
\end{array} \\
& =\frac{\frac{2}{\pi} \varepsilon(r)-\sqrt{1-r^{2}}}{\frac{1}{2}\left[\sqrt{1+r^{2}}+\frac{\sinh ^{-1}(r)}{r}\right]-\sqrt{1-r^{2}}} \\
& =\frac{\frac{4}{\pi} r \varepsilon(r)-2 r \sqrt{1-r^{2}}}{\sinh ^{-1}(r)+\left(r \sqrt{1+r^{2}}-2 r \sqrt{1-r^{2}}\right)} . \\
& \text { Let } f_{1}(r)=\frac{4}{\pi} r \varepsilon(r)-2 r \sqrt{1-r^{2}}, f_{2}(r)=\sinh ^{-1}(r)+\left(r \sqrt{1+r^{2}}-2 r \sqrt{1-r^{2}}\right) \text { and } \\
& f(r)=\frac{\frac{4}{\pi} r \varepsilon(r)-2 r \sqrt{1-r^{2}}}{\sinh ^{-1}(r)+\left(r \sqrt{1+r^{2}}-2 r \sqrt{1-r^{2}}\right)} .
\end{aligned}
$$

Then simple computations lead to

$$
\begin{aligned}
& f_{1}\left(0^{+}\right)=f_{2}(0)=0, \\
& \frac{f_{1}^{\prime}(r)}{f_{2}^{\prime}(r)}=\frac{\frac{2}{\pi} \sqrt{1-r^{2}}[2 \varepsilon(r)-\kappa(r)]+2 r^{2}-1}{2 r^{2}+\sqrt{1-r^{4}}-1}=\frac{\varphi_{1}(r)}{\varphi_{2}(r)},
\end{aligned}
$$

where $\varphi_{1}(r)$ and $\varphi_{2}(r)$ are defined as in Lemmas 2.3 and 2.4.

It follows from Lemmas 2.3-2.4 and (3.7) that $f_{1}^{\prime}(r) / f_{2}^{\prime}(r)$ is strictly increasing on $(0,1)$. Then (3.5), (3.6) and Lemma 2.1 lead to the conclusion that $f(r)$ is strictly increasing.

Moreover,

$$
\begin{aligned}
& \lim _{r \rightarrow 0^{+}} \frac{\frac{4}{\pi} r \varepsilon(r)-2 r \sqrt{1-r^{2}}}{\sinh ^{-1}(r)+\left(r \sqrt{1+r^{2}}-2 r \sqrt{1-r^{2}}\right)}=\frac{3}{8}, \\
& \lim _{r \rightarrow 1^{-}} \frac{\frac{4}{\pi} r \varepsilon(r)-2 r \sqrt{1-r^{2}}}{\sinh ^{-1}(r)+\left(r \sqrt{1+r^{2}}-2 r \sqrt{1-r^{2}}\right)}=\frac{4}{\pi[\pi(\log (1+\sqrt{2})+\sqrt{2})]} .
\end{aligned}
$$

Therefore, Theorem 3.1 follows easily from (3.4), (3.8) and (3.9) together with the monotonicity of $f(r)$.

\section{Theorem 3.2 The double inequality}

$$
\begin{aligned}
\lambda N_{A Q}(a, b)+(1-\lambda) G(a, b) & <T D[A(a, b), G(a, b)] \\
& <\mu N_{A Q}(a, b)+(1-\mu) G(a, b)
\end{aligned}
$$

holds for all $a, b>0$ with $a \neq b$ if and only if $\lambda \leq 3 / 10$ and $\mu \geq 8 /[\pi(\pi+2)]=0.4952 \cdots$.

Proof Without loss of generality, we assume that $a>b>0$ and let $r=(a-b) /(a+b) \in(0,1)$. Then from (1.4) we get

$$
N_{A Q}(a, b)=\frac{1}{2} A(a, b)\left[1+\left(1+r^{2}\right) \frac{\tan ^{-1}(r)}{r}\right]
$$


It follows from (3.2), (3.11) and $G(a, b)=A(a, b) \sqrt{1-r^{2}}$ that

$$
\begin{aligned}
& \frac{T D[A(a, b), G(a, b)]-G(a, b)}{N_{A Q}(a, b)-G(a, b)} \frac{\frac{2}{\pi} \mathcal{E}(r)-\sqrt{1-r^{2}}}{\frac{1}{2}\left[1+\left(1+r^{2}\right) \frac{\tan ^{-1}(r)}{r}\right]-\sqrt{1-r^{2}}} \\
& =\frac{\left[\frac{4}{\pi} r \mathcal{E}(r)-2 r \sqrt{1-r^{2}}\right] /\left(1+r^{2}\right)}{\tan ^{-1}(r)+\left(r-2 r \sqrt{1-r^{2}}\right) /\left(1+r^{2}\right)} .
\end{aligned}
$$

Let $g_{1}(r)=\left[\frac{4}{\pi} r \mathcal{E}(r)-2 r \sqrt{1-r^{2}}\right] /\left(1+r^{2}\right), g_{2}(r)=\tan ^{-1}(r)+\left(r-2 r \sqrt{1-r^{2}}\right) /\left(1+r^{2}\right)$ and

$$
g(r)=\frac{\left[\frac{4}{\pi} r \mathcal{E}(r)-2 r \sqrt{1-r^{2}}\right] /\left(1+r^{2}\right)}{\tan ^{-1}(r)+\left(r-2 r \sqrt{1-r^{2}}\right) /\left(1+r^{2}\right)} .
$$

Then simple computations lead to

$$
\begin{aligned}
& g_{1}\left(0^{+}\right)=g_{2}(0)=0, \\
& \frac{g_{1}^{\prime}(r)}{g_{2}^{\prime}(r)}=\frac{\frac{2}{\pi} \sqrt{1-r^{2}}\left[2 \varepsilon(r)-\left(1+r^{2}\right) \kappa(r)\right]+3 r^{2}-1}{3 r^{2}+\sqrt{1-r^{2}}-1}=\frac{\varphi_{4}(r)}{\varphi_{5}(r)},
\end{aligned}
$$

where $\varphi_{4}(r)$ and $\varphi_{5}(r)$ are defined as in Lemmas 2.6 and 2.7.

It follows from Lemmas 2.6-2.7 and (3.15) that $g_{1}^{\prime}(r) / g_{2}^{\prime}(r)$ is strictly increasing on $(0,1)$. Then (3.13), (3.14) and Lemma 2.1 lead to the conclusion that $g(r)$ is strictly increasing.

Moreover,

$$
\begin{aligned}
& \lim _{r \rightarrow 0^{+}} \frac{\left[\frac{4}{\pi} r \varepsilon(r)-2 r \sqrt{1-r^{2}}\right] /\left(1+r^{2}\right)}{\tan ^{-1}(r)+\left(r-2 r \sqrt{1-r^{2}}\right) /\left(1+r^{2}\right)}=\frac{3}{10}, \\
& \lim _{r \rightarrow 1^{-}} \frac{\left[\frac{4}{\pi} r \varepsilon(r)-2 r \sqrt{1-r^{2}}\right] /\left(1+r^{2}\right)}{\tan ^{-1}(r)+\left(r-2 r \sqrt{1-r^{2}}\right) /\left(1+r^{2}\right)}=\frac{8}{\pi(\pi+2)} .
\end{aligned}
$$

Therefore, Theorem 3.2 follows from (3.12), (3.16) and (3.17) together with the monotonicity of $g(r)$.

From Theorems 3.1-3.2 we get the following Corollary 3.3 immediately.

Corollary 3.3 Let $\alpha=3 / 8, \beta=4 /[\pi(\log (1+\sqrt{2})+\sqrt{2})]=0.5546 \cdots, \lambda=3 / 10$ and $\mu=$ $8 /[\pi(\pi+2)]=0.4952 \cdots$. Then the double inequalities

$$
\begin{aligned}
& \frac{1}{4} \pi \alpha\left[\sqrt{1+r^{2}}+\frac{\sinh ^{-1}(r)}{r}\right]+\frac{1}{2} \pi(1-\alpha) \sqrt{1-r^{2}} \\
& <\mathcal{E}(r)<\frac{1}{4} \pi \beta\left[\sqrt{1+r^{2}}+\frac{\sinh ^{-1}(r)}{r}\right]+\frac{1}{2} \pi(1-\beta) \sqrt{1-r^{2}} \\
& \frac{1}{4} \pi \lambda\left[1+\left(1+r^{2}\right) \frac{\tan ^{-1}(r)}{r}\right]+\frac{1}{2} \pi(1-\lambda) \sqrt{1-r^{2}} \\
& \quad<\mathcal{E}(r)<\frac{1}{4} \pi \mu\left[1+\left(1+r^{2}\right) \frac{\tan ^{-1}(r)}{r}\right]+\frac{1}{2} \pi(1-\mu) \sqrt{1-r^{2}}
\end{aligned}
$$

hold for all $r \in(0,1)$. 


\section{Results and discussion}

In this paper, we provide the sharp bounds for the Toader-type mean in terms of the convex combination of geometric and Neuman means. As applications, we find new bounds for the complete elliptic integral of the second kind.

\section{Conclusion}

In the article, we present the optimal convex combination bounds of the geometric and Neuman means for the Toader-type mean, and give several new upper and lower bounds for the complete elliptic integral of the second kind. The given results are the improvements of some previously known results.

\section{Acknowledgements}

This research was supported by the Natural Science Foundation of Zhejiang Province under Grant LY13A010004 and the Natural Science Foundation of Zhejiang Broadcast and TV University under Grant XKT-15G17.

\section{Competing interests}

The authors declare that they have no competing interests.

\section{Authors' contributions}

All authors contributed equally to the writing of this paper. All authors read and approved the final manuscript.

\section{Author details}

${ }^{1}$ School of Mechanical and Electrical Engineering, Huzhou Vocational \& Technical College, Huzhou, 313000, China

${ }^{2}$ School of Distance Education, Huzhou Broadcast and TV University, Huzhou, 313000, China.

\section{Publisher's Note}

Springer Nature remains neutral with regard to jurisdictional claims in published maps and institutional affiliations.

Received: 7 May 2017 Accepted: 14 August 2017 Published online: 29 August 2017

\section{References}

1. Carlson, BC: Special Functions of Applied Mathematics. Academic Press, New York (1977)

2. Toader, GH: Some mean values related to the arithmetic-geometric mean. J. Math. Anal. Appl. 218(2), 358-368 (1998)

3. Neuman, E, Sándor, J: On the Schwab-Borchardt mean. Math. Pannon. 14(2), 253-266 (2003)

4. Neuman, E, Sándor, J: On the Schwab-Borchardt mean II. Math. Pannon. 17(1), 49-59 (2006)

5. Neuman, E: Inequalities for the Schwab-Borchardt mean and their applications. J. Math. Inequal. 5(4), $601-609$ (2011)

6. Neuman, E: On a new bivariate mean. Aequ. Math. 88(3), 277-289 (2014)

7. Neuman, E: Bounds for symmetric elliptic integrals. J. Approx. Theory 122(2), 249-259 (2003)

8. Kazi, H, Neuman, E: Inequalities and bounds for elliptic integrals. J. Approx. Theory 146(2), 212-226 (2007)

9. Kazi, H, Neuman, E: Inequalities and bounds for elliptic integrals II. In: Special Functions and Orthogonal Polynomials. Contemp. Math., vol. 471, pp. 127-138. Am. Math. Soc., Providence (2008)

10. Chu, Y-M, Wang, M-K, Qiu, S-L, Qiu, Y-F: Sharp generalized Seiffert mean bounds for Toader mean. Abstr. Appl. Anal. 2011, Article ID 605259 (2011)

11. Chu, Y-M, Wang, M-K: Inequalities between arithmetic-geometric, Gini, and Toader means. Abstr. Appl. Anal. 2012, Article ID 830585 (2012)

12. Chu, Y-M, Wang, M-K: Optimal Lehmer mean bounds for the Toader mean. Results Math. 61(3-4), 223-229 (2012)

13. Chu, Y-M, Wang, M-K, Qiu, S-L: Optimal combination bounds of root-square and arithmetic means for Toader mean. Proc. Indian Acad. Sci. Math. Sci. 122(1), 41-51 (2012)

14. Hua, Y, Qi, F: The best bounds for Toader mean in terms of the centroidal and arithmetic means. Filomat 28(4), 775-780 (2014)

15. Song, Y-Q, Jiang, W-D, Chu, Y-M, Yan, D-D: Optimal bounds for Toader mean in terms of arithmetic and contraharmonic means. J. Math. Inequal. 7(4), 751-757 (2013)

16. Li, W-H, Zheng, M-M: Some inequalities for bounding Toader mean. J. Funct. Spaces Appl. 2013, Article ID 394194 (2013)

17. Sun, H, Chu, Y-M: Bounds for Toader mean by quadratic and harmonic means. Acta Math. Sci. 35A(1), 36-42 (2015) (in Chinese)

18. Hua, Y, Qi, F: A double inequality for bounding Toader mean by the centroidal mean. Proc. Indian Acad. Sci. Math. Sci. 124(4), 527-531 (2014)

19. Chu, Y-M, Wang, M-K, Ma, X-Y: Sharp bounds for Toader mean in terms of contraharmonic mean with applications. J. Math. Inequal. 7(2), 161-166 (2013)

20. Zhao, T-H, Chu, Y-M, Zhang, W: Optimal inequalities for bounding Toader mean by arithmetic and quadratic means. J. Inequal. Appl. 2017, Article ID 26 (2017)

21. Anderson, GD, Vamanamurthy, MK, Vuorinen, M: Conformal Invariants, Inequalities, and Quasiconformal Maps. Wiley, New York (1997) 
22. Wang, M-K, Li, Y-M, Chu, Y-M: Inequalities and infinite product formula for Ramanujan generalized modular equation function. Ramanujan J. doi:10.1007/s11139-017-9888-3

23. Wang, M-K, Chu, Y-M: Refinements of transformation inequalities for zero-balanced hypergeometric functions. Acta Math. Sci. 37B(3), 607-622 (2017)

24. Chu, H-H, Qian, W-M, Chu, Y-M, Song, Y-Q: Optimal bounds for a Toader-type mean in terms of one-parameter quadratic and contraharmonic meas. J. Nonlinear Sci. Appl. 9(5), 3424-3432 (2016)

25. Song, Y-Q, Zhao, T-H, Chu, Y-M, Zhang, X-H: Optimal evaluation of a Toader-type mean by power mean. J. Inequal. Appl. 2015, Article ID 408 (2015)

26. Qian, W-M, Song, Y-Q, Zhang, X-H, Chu, Y-M: Sharp bounds for Toader mean in terms of arithmetic and second contraharmonic means. J. Funct. Spaces 2015, Article ID 452832 (2015)

27. Wang, M-K, Chu, Y-M, Qiu, Y-F, Qiu, S-L: An optimal power mean inequality for the complete elliptic integrals. Appl. Math. Lett. 24(6), 887-890 (2011)

28. Chu, Y-M, Wang, M-K, Qiu, Y-F: On Alzer and Qiu's conjecture for complete elliptic integral and inverse hyperbolic tangent function. Abstr. Appl. Anal. 2011, Article ID 697547 (2011)

29. Wang, M-K, Qiu, S-L, Chu, Y-M, Jiang, Y-P: Generalized Hersch-Pfluger distortion function and complete elliptic integrals. J. Math. Anal. Appl. 385(1), 221-229 (2012)

30. Wang, M-K, Chu, Y-M, Qiu, S-L, Jiang, Y-P: Convexity of the complete elliptic integrals of the first kind with respect to Hölder means. J. Math. Anal. Appl. 388(2), 1141-1146 (2012)

31. Chu, Y-M, Wang, M-K, Qiu, S-L, Jiang, Y-P: Bounds for complete elliptic integrals of the second kind with applications. Comput. Math. Appl. 63(7), 1177-1184 (2012)

32. Chu, Y-M, Wang, M-K, Jiang, Y-P, Qiu, S-L: Concavity of the complete elliptic integrals of the second kind with respect to Hölder means. J. Math. Anal. Appl. 395(2), 637-642 (2012)

33. Chu, Y-M, Qiu, Y-F, Wang, M-K: Hölder mean inequalities for the complete elliptic integrals. Integral Transforms Spec. Funct. 23(7), 521-527 (2012)

34. Wang, M-K, Chu, Y-M: Asymptotical bounds for complete elliptic integrals of the second kind. J. Math. Anal. Appl. 402(1), 119-126 (2013)

35. Chu, Y-M, Qiu, S-L, Wang, M-K: Sharp inequalities involving the power mean and complete elliptic integral of the first kind. Rocky Mt. J. Math. 43(5), 1489-1496 (2013)

36. Wang, G-D, Zhang, Z-H, Chu, Y-M: A power mean inequality involving the complete elliptic integrals. Rocky Mt. J. Math. 44(5), 1661-1667 (2014)

37. Yang, Z-H, Chu, Y-M, Zhang, W: Accurate approximations for the complete elliptic integral of the second kind. J. Math Anal. Appl. 438(2), 875-888 (2016)

38. Yang, Z-H, Chu, Y-M, Zhang, X-H: Sharp Stolarsky mean bounds for the complete elliptic integral of the second kind. J. Nonlinear Sci. Appl. 10(3), 929-936 (2017)

39. Yang, Z-H, Chu, Y-M, Zhang, W: Monotonicity of the ratio for the complete elliptic integral and Stolarsky mean. J. Inequal. Appl. 2016, Article ID 176 (2016)

40. Yang, Z-H, Chu, Y-M, Wang, M-K: Monotonicity criterion for the quotient of power series with application. J. Math. Anal. Appl. 428(1), 587-604 (2015)

41. Wang, M-K, Wang, Z-K, Chu, Y-M: An optimal double inequality between geometric and identric means. Appl. Math. Lett. 25(3), 471-475 (2012)

42. Vuorinen, M: Hypergeometric functions in geometric function theory. In: Special Functions and Differential Equations, Madras, 1997, pp. 119-126. Allied Publ., New Delhi (1998)

43. Qiu, S-L, Shen, J-M: On two problems concerning means. J. Hangzhou Inst. Electron. Eng. 17(3), 1-7 (1997) (in (hinese)

44. Barnard, RW, Pearce, K, Richards, KC: An inequality involving the generalized hypergeometric function and the arc length of an ellipse. SIAM J. Math. Anal. 31(3), 693-699 (2000)

45. Alzer, H, Qiu, S-L: Monotonicity theorems and inequalities for the complete elliptic integrals. J. Comput. Appl. Math. 172(2), 289-312 (2004)

46. Li, J-F, Qian, W-M, Chu, Y-M: Sharp bounds for Toader mean in terms of arithmetic, quadratic, and Neuman means. J. Inequal. Appl. 2015, Article ID 277 (2015)

\section{Submit your manuscript to a SpringerOpen ${ }^{\circ}$ journal and benefit from:}

- Convenient online submission

- Rigorous peer review

- Open access: articles freely available online

- High visibility within the field

- Retaining the copyright to your article

Submit your next manuscript at $\gg$ springeropen.com 